\title{
Subject Index Vol. 40, 1997
}

Aneurysm 13 Athetosis 26,124,135

Bilateral cervical posterior rhizotomy

Blood-brain banier 72

Bram stem reticular formation 26

Caudate nucleus 48 Cerebellar 141

- stimulation $124,135,160$

Cerebellum 124

Cerebral palsy $124,135,160$

Chronic stimulation 223

Clinical results 111

Complications 218

Computers 88

Conus medullaris 192 Current damage 141

- density 141

Demyelinating disease 208

Dorsal column stimulation 184, 208

Dystonia 26, 160

\section{EEG 88}

Electrical stimulation 235

- $\quad$ - of neural tissue 72

Electrode arrays 72

Electrodes 72, 88,141

Endorphin 111

Epilepsy 48, 88,124

ESB 88

Evoked potentials 141, 160

Footdrop 235 D?oreI-H-tomy 1

Hippocampus 48

Implants 141 Intracranial 13 Intractable epilepsy 1 Involuntary movements 160

Learning 88

Lumbar myelotomy 41 
Medical instrumentation 218

- device 175, 218

Microprocessor 88

Movement 88

Multiple sclerosis 160, 208

Nerve implant 223

- lesion 111 Neural damage 72

- prostheses 72 Neuroaugmentation 175, 218 Neurogenic bladder 192

Neuroprosthesis 235 Neurostimulation 88,175, 218 Neurosurgery 13

Pain 88,111,175,184,223 Paraplegic 192 Percutaneous electrodes 184

Peripheral nerve 235

- - stimulation 223

- nerves 223 Potassium chloride 48

Radiology 13

Sejective postenor rhizotomy 41 Sleep-induced apnea 26 Slow potential recording 48 Spasticity 41, 124, 135, 160 Spinal cord 184

- - stimulation 192 Spinocerebellar ataxia 208 Stereotactic 13

- surgery 48

- - for epilepsy 1 Stereotaxis 111 Stimulation 184 Stimulator 124

Stimulators 88

Telemetry 88 Thalamic syndrome 111 Thalamus 111

Vascular disease 208 